\title{
ELECTRON LINACS IN RADIOACTIVE WASTE DISPOSAL PROBLEM*
}

\author{
N.P. Dikiy, A.N. Dovbnya, S.Yu. Sayenko, V.L. Uvarov*** \\ National Science Center "Kharkov Institute of Physics \& Technology” (KIPT),61108, Kharkov, \\ Ukraine
}

\begin{abstract}
Development of nuclear technologies is accompanied by the growth of radioactive waste including long-lived ones. The waste inside the Chernobyl 4-th unit is of particular importance because their amount is estimated as much as $20 \mathrm{MCi}$. Under the circumstances a problem of operative characterization of the waste and their longterm disposal is urgent. The report presents an overview of the waste characterization method elaborated in NSC KIPT based on $\gamma$-activation analysis using bremsstrahlung of the high-current electron linac. On the other hand disposal of the radioactive waste faces a problem of confinement materials (including geological structures). Such materials have to keep their protection properties with respect to radionucliede transport under absorbed dose value up to $\sim 10^{7}$ Gy during thousand years or so. The elaborated methods for production of radionucliedetracers and operative determination of their diffusion coefficients into barriers under different doses of the braking photons are described.
\end{abstract}

\section{INTRODUCTION}

A handling with radioactive waste (RAW) includes a number of procedures. First of all it is their characterization i.e. determination of the amount, activity, radionuclide and element content etc. The disposable methods of RAW characterization are based either on analysis of their inherent radiation $(\gamma-, \beta-, \alpha-$ spectrometry) or on the profound radiochemical treatment of the samples with an extraction of corresponding analysed fraction for its further spectrometry. The shortcomings of the first group methods are relatively low accuracy and a restriction to the analysis of only thin RAW layer (particularly $\beta$ - and $\alpha$-active ones) as well as a small number of identifiable elements. Second group methods are devoid of these shortcomings. However they are rather labor-consuming, expensive and low operative (a duration of one radiochemical analysis is up to several days). It is known that the activation method based on electron accelerators secondary radiation is widely used at present for indestructive express-analysis of the ore and different materials samples [1], fission materials [2] and in other fields.

Taking into account that a RAW sample activated by high-energy braking photons emits a radiation that is caused both by its inherent activity and initiated one as a result of photonuclear reactions, then an analysis of such radiation gives quantitative information about radionuclide and element composition of the specimen without its dissection.

The next RAW handling stage is their immobilization and disposal in the steady geological structures. This task calls elaboration of experimental prognostication methods for lasting (up to thousand years) conduct of the disposal environment under complicated radiation and corrosive conditions.

\section{ACCELERATOR}

1.1. For the analysis of large amount of the RAW samples by means of $\gamma$-activation method and implementation of other concomitant programs it is needed an electron accelerator with beam power up to 10 $\mathrm{kW}$ and a wide range of particle energy regulation.

The complex LU-20 [3] designed in "Accelerator" R\&D Prod. Est. of NSC KIPT satisfies these requirements (see Table 1).

Table 1. Basic parameters of LU-20 Linac

$\begin{array}{lll}\text { Energy range, } \mathrm{MeV}- & - & 10 \ldots . .30 \\ \text { Pulse duration, } \mu \mathrm{s} & - & 4 \\ \text { Maximum repetition rate, } \mathrm{Hz} & - & 300 \\ \text { Maximum peak current, } \mathrm{mA} & - & 1000 \\ \text { Maximum average current, } \mu \mathrm{A} & - & 1000 \\ \begin{array}{l}\text { Beam scanning frequency, } \mathrm{Hz} \\ \text { Beam size at the accelerator }\end{array} & - & 3 \\ \quad \begin{array}{l}\text { exit, cm } \\ \text { Absorbed dose rate }\end{array} & - & 2 \times 30 \\ \quad \text { (electrons), Gy/h } & - & \text { up to } 4 \cdot 10^{7} \\ \text { Absorbed dose rate } & & \\ \text { (braking photons), Gy/h } & - & \text { up to } 1 \cdot 10^{5}\end{array}$

1.2. A necessary set of radiation forming and diagnostics devices has been developed for ensuring $\gamma$ activation analysis (Fig.1).

An electron beam at the accelerator $\mathbf{A}$ exit is scanned using electromagnet SM. A continuous beam current monitoring is carried out by the magnetoinductive sensor MIS and linear beam coordinate using beam position monitor BMP [4]. A converter assembly $\mathbf{C}$ consists of tantalum plate that is placed into aluminium casing and is cooled by running water. The filter $\mathbf{F}$ (5 aluminium plates) absorbs the part of the electron beam that passed the converter assembly. A braking photon flux after filter F measuring typical dimensions $150 \times 500 \mathrm{~mm}$ is controlled by wide- aperture ionization chamber IC-W [5]. The capsules with analysed specimens are placed just behind the IC-W.

\footnotetext{
* Work is supported by STCU under contract N 1580

** E-mail: uvarov@kipt.kharkov.ua
} 


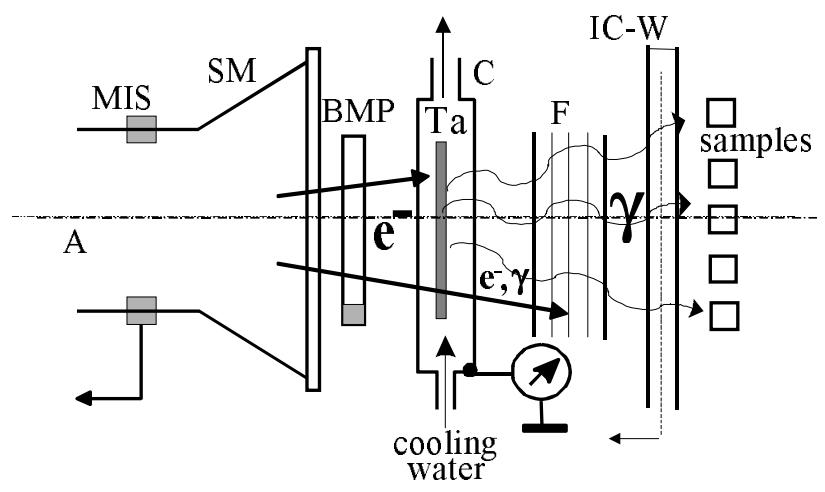

Fig.1. Schematic of the radiation forming and diagnostics devices

In case when electron energy exceeds $10 \mathrm{MeV}$ an isotropic stream of photoneutrons is emitted from the converter together with braking photons. These neutrons can be used also in the framework of considered problem.

\section{RAW ANALYSIS}

An investigated RAW sample is irradiated as a rule together with a specimen of the standard isotope content. A concentration of this isotope in the sample is determined by means of the comparison of the induced $\gamma$ activity of each sample along the lines corresponding to given isotope (taking into account the mass of the specimen).

As an example, Fig.2,3 show the induced $\gamma$-spectrum for two samples of materials of the wrecked 4-th Unit of Chernobyl station: fragments of the reactor concrete shield (Fig.2) and lava-like fuel-containing mass (LFCM), which was formed in underreactor premises as a result of the accident (Fig.3).

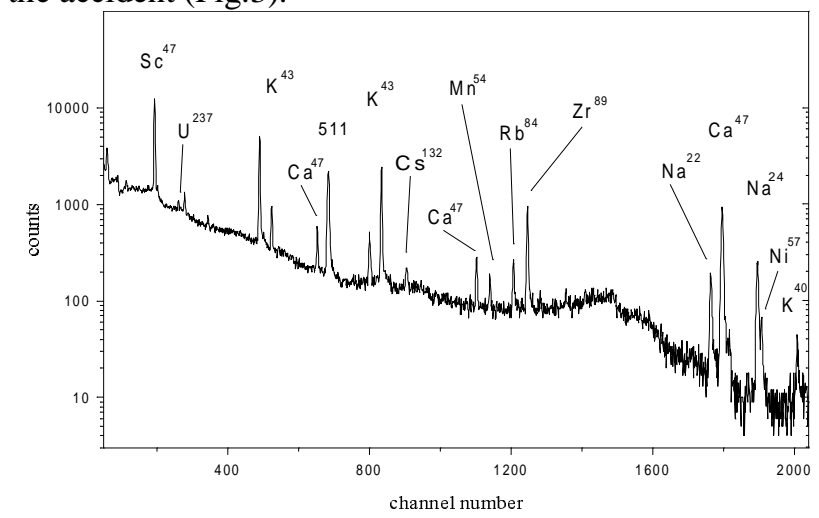

Fig.2. Induced $\gamma$-activity spectrum of concrete sample

The spectrum in Fig.2 includes the U-237 line. This isotope was generated in the sample under activation process in ${ }^{238} U(\gamma, n){ }^{237} U$ reaction. This example demonstrates the ability of the $\gamma$-activation method to analyse the elements which identification is impossible by means of traditional spectrometry methods. Thus obtained quantitative data concerning the element content in the samples allow to carry out a correlation analysis as well.

Fig.3 demonstrates also the ability of $\gamma$-activation method in analysis of the samples having their own activity of different nature.

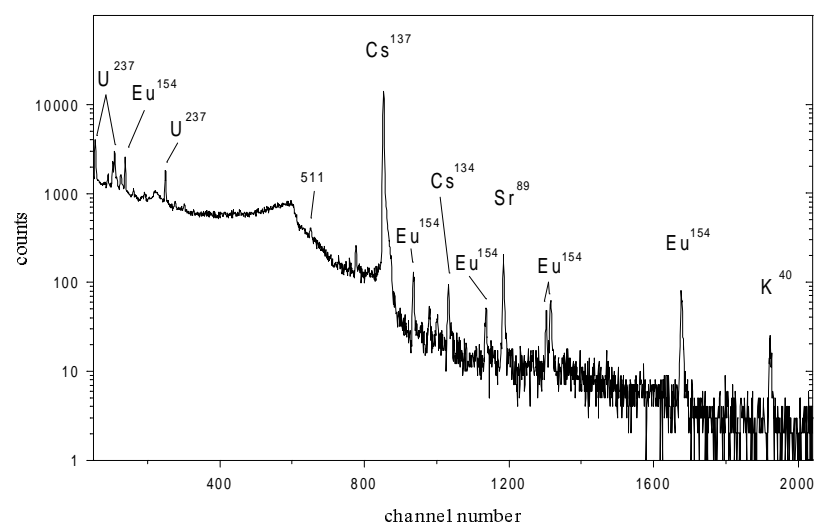

Fig.3. Spectrum of activated LFCM sample

So, apart the lines of $\gamma$-radiating nuclides (Eu-154, Cs-137 and Cs-134) there are shown the lines of U-237 and Sr89. The last result is especially important because $\gamma$ radiating nuclide $\mathrm{Sr}-89$ is created as a consequence of the $\beta$-radiating Sr-90 activation. It is known that an analysis of the $\gamma$-radiating nuclides is realized technically simpler and for more thick RAW layers (up to $30 \mathrm{~cm}$ or so).

Spectrum on Fig.4 corresponds to activated U-238 dioxide water solution (with concentration $30 \mathrm{mg}$ U238/l). These data show that $\gamma$-activation method can be used also for the analysis of liquid RAW with identification limit not more then $2 \mu \mathrm{g} \mathrm{U}-238 / l$.

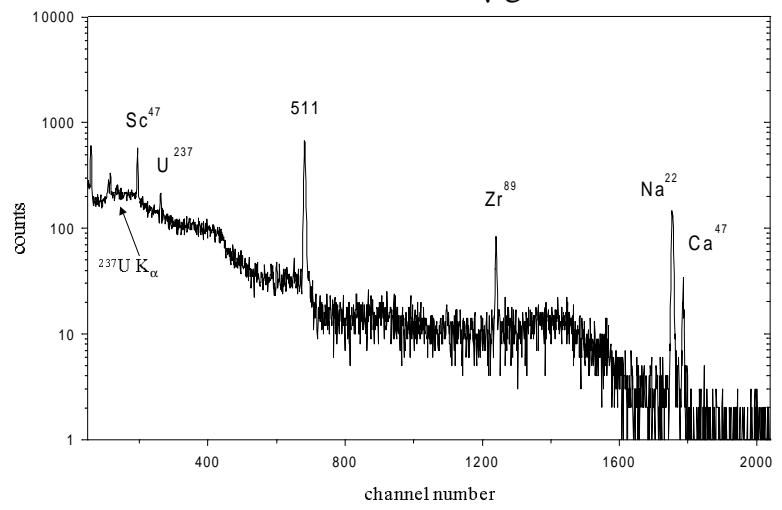

Fig.4. $\gamma$-spectrum of activated U-238 water solution

\section{INVESTIGATION OF MATERIALS FOR RAW DISPOSAL}

It is known that during lasting disposal of the highlevel RAW or nuclear spent fuel can arise a situation when the RAW immobilization matrix (including geological structure) will contact with ground water. Thus originates a structure of "RAW-water-geological barrier" type. A radionuclide transport in such structure determines a reliability of the RAW disposal. Such transport depends besides all on absorbed dose of radiation from the RAW estimated as much as $10^{8} \mathrm{~Gy}$ during disposal period.

For research of radionuclide transport processes the granite specimens (which is considered as a perspective environment for disposal of long-lived RAW) were selected. A piece of granite was cut into the specimens in the form of blocks with the size of $10 \times 10 \mathrm{~mm}$ in cross- 
section and $30 \mathrm{~mm}$ in thickness. Each block was covered with epoxy except for 10x10 surface.

Isotope $\mathrm{Yb}-169$ was used as $\gamma$-radiating nuclide-tracer which is analogous to actinides in its chemical properties. For this nuclide production under reaction ${ }^{168} \mathrm{Yb}(\mathrm{n}$, $\gamma)^{169} \mathrm{Yb}$ the pellets of stable ${ }^{168} \mathrm{Yb}_{2} \mathrm{O}_{3}$ were irradiated by photoneutrons. Then the pellet was dissolved in concentrated $\mathrm{HCl}$ acid $(0.2 \mathrm{ml})$ and finally the aqueous solution with $\mathrm{pH}=1.8$ was prepared.

Obtained solution $(40 \mathrm{ml})$ together with specimen irradiated up to given dose value $\left(3 \cdot 10^{6} \ldots 3 \cdot 10^{7} \mathrm{~Gy}\right)$ were placed into thermostable flask. The latter was being heated by water steam during 32 hours.

Then each specimen was being washed in distillate water during 24 hours and dried out at $60^{\circ} \mathrm{C}$ in the drying box. Further the layers $(2 . .50 \mu \mathrm{m})$ from free surface of the specimen were removed by means of precision grinding. Material of the removed layers was used for $\gamma$ spectrometry with the $\mathrm{Ge}(\mathrm{Li})$-detector. Typical spectrum of removed material of the sample, irradiated with braking photons of LU-20 accelerator up to dose value 3 $10^{7} \mathrm{~Gy}$ is shown in Fig.5. Spatial distribution of Yb-169 concentration within depth of the specimen is demonstrated in Fig.6. These results allowed to determine a dose dependence of the radionuclide diffusion as well as to find out its mechanism [7].

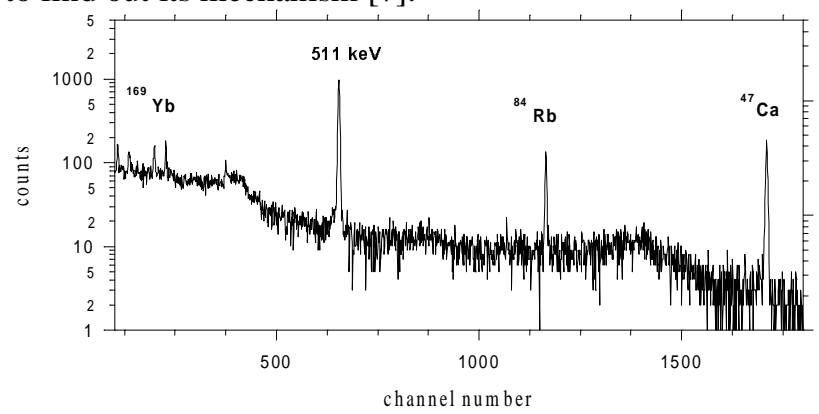

Fig.5. $\gamma$-spectrum of irradiated granite $\left(3 \cdot 10^{7} \mathrm{~Gy}\right)$

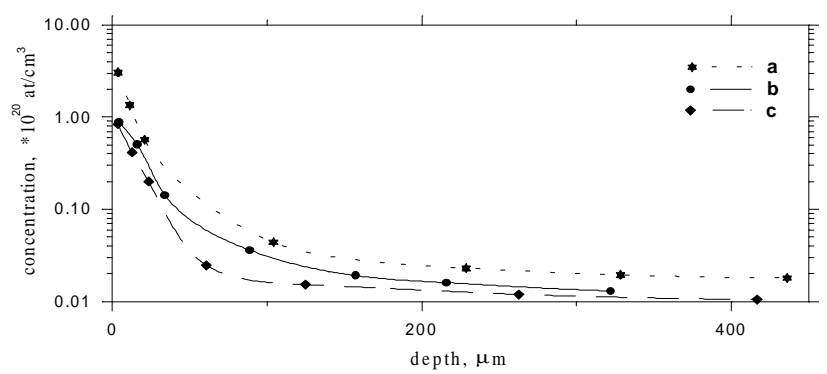

Fig.6. Distribution of Yb-169 into granite: a - irradiated $\left(3 \cdot 10^{7} \mathrm{~Gy}\right)$;

b - pristine state (granite with pegmatite structure);

c - pristine state (granite with uniform grain structure)

\section{COMPUTER SIMULATION}

A method of the computer simulation on base of standardized code GEANT (or other like it) can be used for optimization of the sample irradiation conditions, isotope generation modelling as well as for investigation of metrological characteristics of the measuring sensors under their interaction with radiation. Such code allows to calculate these parameters with appropriate accuracy (not less $10 \%$ ) considering real composition of the radiation forming systems as well as of the irradiated object [8].

\section{CONCLUSIONS}

1. High-current electron accelerator with energy range $10 \ldots 30, \mathrm{MeV}$ allows to solve effectively different problems of radioactive waste handling, in particularly, operative analysis of the nuclide and element content of the RAW using $\gamma$-activation method without dissection of the samples. This method provides also an ability of distant-reading analysis under automatic operation. Such facility is important for large amount of the analysed samples, for example, when extracting the RAW from the 4-th Chernobyl unit.

2. A powerful $(\sim 10 \mathrm{~kW})$ electron accelerator in its absorbed dose rate ability is comparable with Co-60 source having activity up to $1 \mathrm{MCi}$. This circumstance as well as a possibility to control upper limit of the braking photons spectrum allow to use linacs for research of radiation \& chemical stability of the materials intended for immobilization and disposal of the RAW. The radionuclides produced directly on linac can be used as tracers in these investigations.

3. Linac provides the radiation of different intensity and nature (accelerated electrons, bremsstrahlung and photoneutrons) that gives a possibility of radiation test of materials within the wide range of their operation conditions.

\section{REFERENCES}

[1] Toms M. Elaine. Photonuclear Activation Analysis with $\mathrm{Ge}(\mathrm{Li})$ Detector. Nav. Res. Lab., USA, Rep. 7554, 1973.

[2] T. Gozani et al. Measurement of Prompt and Delayed Neutrons from Photofission. ANS Trans., 1968, v. 11, p. 659.

[3] A.N. Dovbnya et al. Electron Linacs Based Radiation Facilities of Ukrainian National Science Center "KIPT". Bul. of the Amer. Phys. Soc., 1997, v. 42, N 3, p. 1391.

[4] V.L. Uvarov, V.N.Boriskin et al. Calibration of Electron Beam Measuring in Technological Linacs, ICALEPS'99, Trieste, Italy, 1999.

[5] A.A. Butenko, S.P. Karasyov et al. Technological Measuring Channel for Bremsstrahlung Monitoring. Voprosy Atomnoj Nauki i Techniki (in russian), 1999, v. 4(35), p. 49.

[6] A. Borovoi. Post-Accident Management of Destroyed Fuel from Chernobyl: Technologies Used and Lessons Learned, IAEA, 1990:15.

[7] N.P. Dikiy, S.Yu. Sayenko, V.L. Uvarov, E.P. Shevyakova. Application of Nuclear-Physics Methods for Studying the Radionuclide Transport in Granite Rocks. Voprosy Atomnoj Nauki i Techniki (in russian), 2000, v. 2(36), p. 54.

[8] S.P. Karasyov, S.V. Maryokhin, V.L. Uvarov et al. On Computer Modelling of Primary Transducers in Electron Radiation. Diagnostics, EPAC'98, Stockholm, June 1998, p. 134. 